\title{
Clinical Aspects of Skeletal Muscle Modulators in Type 2 Diabetes Mellitus
}

\author{
Mariusz Henryk Madalinski and Leszek Kalinowski
}

Additional information is available at the end of the chapter

http://dx.doi.org/10.5772/47875

\section{Introduction}

Isotonic and isometric striated muscles contraction, as well as their extensibility and elasticity, are modulated by the central nervous system. Modulation at the level of neuromuscular synapses is also very compound, but precisely regulated, and essential for the induction of signals to muscle cells (Farahat \& Herr 2010). The induced signal starts a cascade of multiple processes, necessary for sustaining muscle homeostasis. Without it, and also without muscle fuel utilization, muscle movement and skeletal support are deeply impaired. We therefore begin our description with electrochemical transmission, because its effects are subjected to modulation.

\section{Calcium and ATP - mediators/modulators of ion channels in myocyte and its contractile activity}

When a threshold potential is reached, an impulse travels along the muscle cell membrane and opens calcium gates in the membrane of the sarcoplasmic reticulum (SR). This is a trigger for the myofibrils and myofilaments located in the sarcoplasm, which turns the muscle "on". A muscle stops contracting, if the impulse is no longer being transferred from the neuron to the sarcolemma. At that moment, calcium gates close and calcium ions move back into the $\mathrm{SR}$. At the terminal cisternae of SR with intracellular $\mathrm{Ca}^{2+}$ stores, the ryanodine receptor channel isoform 1 (RyR1) is located. It is an integral component of excitation-contraction coupling and the receptor activation plays a key role in this process. Excitation-contraction coupling may be triggered by a single-pulse of electrical stimulation and results in massive release of $\mathrm{Ca}^{2+}$ from the SR via the RyR1 to the cytosol after depolarization of the muscle plasma membrane. However, skeletal muscle function in vivo is generally regulated during repetitive firing of short trains of action potentials (Diaz-Sylvester et al., 2008). 
The free cytosolic $\mathrm{Ca}^{2+}$ concentration in a skeletal muscle at rest is about $120 \mathrm{nM}$ and in the millimolar range in its intracellular stores (Eltit et al., 2011). The RyR1 is activated by an increase in cytosolic $\mathrm{Ca}^{2+}$ levels (with peak activation at $\sim 100 \mu \mathrm{M}$ ); regardless of the presence or absence of $\mathrm{Mg}^{2+} / \mathrm{ATP}$, and it is called calcium induced calcium release mechanism. A millimolar $\mathrm{Ca}^{2+}$ concentration also inactivates per se the RyR1. The receptor may also be inhibited by $\mathrm{Mg}^{2+}$ or activated by caffeine and ATP. The RyR1 channel is furthermore modulated by ryanodine, calmodulin, $12-\mathrm{kDa}$ FK-506-binding protein (FKBP12) and socalled Homer proteins, as well as by various redox processes (Diaz-Sylvester et al., 2008; Eltit et al. 2011; Feng et al., 2008; Ye et al., 2012; Lawler et al., 2010).

Variations in the intracellular levels of endogenous RyR1 modulators are relevant to individuals with genetic disorders of skeletal muscles associated with mutations in the RyR1 of SR. Some of the disorders were investigated in malignant hyperthermia (MH) provoked by halothane. The RyR1 becomes more susceptible to activation by halothane, when $\mathrm{Mg}^{2+}$ levels decrease and/or $\mathrm{Ca}^{2+}$ levels increase in the cytosol. ATP binding greatly increases the RyR1 activity as well. Moreover, halothane-induced RyR1 activation is enhanced by increases in SR $\mathrm{Ca}^{2+}$ loads. Knowledge about cytosolic modulators may help us understand, why decreased $\mathrm{Mg}^{2+}$ sensitivity may result in higher sensitivity to halothane and why RyR1 carrying $\mathrm{MH}$ mutations are less sensitive to $\mathrm{Mg}^{2+}$ inhibition. Taking into account the fact that insulin decreases intracellular $\mathrm{Mg}^{2+}$ in muscles, we are able to explain why $\mathrm{MH}$ susceptibility has been associated with increased insulin levels (Diaz-Sylvester et al., 2008).

Another calcium channel is the dihydropyridine receptor (DHPR), serving as a voltage sensor in excitation-contraction coupling and a modulator of RyR1 through physical interactions (a conformational change of DHPR allosterically activates RyR1). In continuously depolarized skeletal muscle fibers, DHPR voltage sensors are inactivated and unable to initiate SR $\mathrm{Ca}^{2+}$ release. Both DHPR and RyR1 receptors bind with Triadin (a modulator from a family of proteins of the junctional sarcoplasmic reticulum) that is involved in many crucial aspects of muscle functions and E-C coupling in skeletal muscles (Diaz-Sylvester et al. 2008; Perez, 2011). Dihydropyridine-receptor agonists and antagonists can modulate both a single pulse electrical stimulation (which mainly triggers a fast calcium signal sensitive to RyR1) and tetanic stimulation (which induces a long-lasting, inositol triphosphate ( $\left.\mathrm{IP}_{3}\right)$-generated signal) (Lawler et al., 2010).

$\mathrm{Ca}^{2+}$ release plays an essential role in initiating muscle contraction, increases glucose uptake (although its signalling is unresolved), activates numerous genes required for mitochondrial biogenesis, and results in ATP production in mitochondrial and cytoplasmic compartments during exercise (Hawley et al. 2006). A lasting contraction causes energy expenditure and ATP supply diminishes at some point (but usually no more than about $20 \%$ of it even during very intensive exercise). This decline in ATP level causes muscle fatigue. The myosin heads remain bound to actin and can no longer swivel. Even when a nervous impulse is being transmitted to the muscle and calcium ions are still present, contraction/or strong contraction is not possible. Several different energy sources (phosphocreatine, muscle glycogen, blood glucose and fatty acids from adipose tissue) are used by myocytes to 
maintain ATP levels in a working muscle. Although glucose is a predominant fuel for the muscles, diminished ATP levels in the myocyte are a signal to switch to the use of fat fuel, leading to an increased flux of free fatty acids and changes in the AMP/ATP ratio, contributing to fiber shifts. Fuel economy depends on the type of muscle fibres involved, the length of the physical effort and its type. There are high speed fibres which require carbohydrate fuels and generally used slowly contracting economical fibers with a fat-based aerobic metabolism (de Lange et al., 2007).

The AMP/ATP ratio is important for the activation of AMP-activated protein kinase (AMPK), which stimulates energy generating processes (glucose uptake and fatty acid oxidation). AMPK plays a key role in controlling muscle economy, inmaintaining muscle homeostasis, and in inducing a cascade of events within cells in response to the everchanging energy charge of the cell (de Lange et al., 2007; Lira et al. 2010).

\section{Modulators of the AMP-activated protein kinase relevant in type 2 diabetes mellitus}

AMPK was discovered in skeletal muscles in 1995 (Verhoeven et al., 1995). It is a heterotrimeric enzyme comprised of two catalytic ( $\alpha 1$ or $\alpha 2)$ subunits and two regulatory ( $\beta 1$ or $\beta 2$ and gamma1, gamma2, or gamma3) subunits (all are encoded by separate genes). AMPK activity inhibited preparations of acetyl-CoA carboxylase (ACC) and 3-hydroxy-3methylglutaryl-CoA reductase (HMG-CoA reductase) was first discovered. Later, it was noticed that AMPK activity can also be regulated by physiological stimuli, independent of the energy charge of the cell (like hormones and nutrients) (Lim et al., 2010; Steinberg \& Kemp, 2009).

AMPK is activated during contraction. Its modulation (related to the muscle energy state) depends on two distinct mechanisms - on an AMP-dependent pathway mediated by kinase LKB1 and on a $\mathrm{Ca}^{2+-}$ dependent pathway mediated by the calmodulin-dependent protein kinase $(\mathrm{CaMKKb})$. AMPK in skeletal muscles increases fatty acid oxidation and mitochondrial biogenesis (Lim et al., 2010). These processes require phosphorylation of the peroxisome proliferator-activated receptor- $\gamma$ coactivator $1 \alpha$ (PGC-1 $\alpha$ ) and SIRT1. The last protein works as an $\mathrm{NAD}^{+}$-dependent protein deacetylase or ADP-ribosyltransferase. It also activates PGC-1 $\alpha$ involved in fatty acid utilization and mitochondrial respiration (de Lange et al., 2007; Zhang et al., 2009).

Mitochondrial dysfunction observed in type 2 diabetes mellitus leads to a decrease in ATP levels and possibly to changes in $\mathrm{NAD}^{+} / \mathrm{NADH}$ levels. It can influence methylenetetrahydrofolate dehydrogenase $\left(\mathrm{NADP}^{+}\right)$activity, which depends on the concentration of $\mathrm{NAD}^{+}$and on the ATP/ADP ratio (Zhang et al., 2009; Mailloux \& Harper, 2010). NADH inhibits and $\beta$ NAD activates AMPK, but NAD is a much weaker activator than AMP (we also do not know, if AMP is a direct activator). AMPK activity is modulated not only by nucleotides, but also by kinases, fatty acids, insulin, cytokines and reactive oxygen species (ROS) (Rafaeloff-Phail et al., 2004; Steinberg \& Kemp, 2009; Zhang et al., 2009). 
The major glucose transporter - GLUT4 translocation to sarcolemma, is also a result of AMPK-activated PGC- $1 \alpha$, where SIRT1 activity is required (Steinberg \& Kemp, 2009). This pathway of glucose uptake in skeletal muscles is stimulated by palmitate (PA) (regardless of AMP concentrations) (Pu et al., 2011). It takes place only when skeletal muscles are exposed to PA for a short time. Chronic exposure to PA blocks Akt phosphorylation and inhibits insulin induced glucose uptake. Besides PA, linoleic, oleic, and stearic acids can stimulate AMPK and Akt (Pu et al., 2011).

5-Aminoimidazole-4-carboxyamide ribonucleoside (AICAR) is another AMPK activator increasing GLUT4 translocation to sarcolemma and glucose uptake in rats' skeletal muscles. AICAR as well as physical effort, leads to AMPK activation, which enhances a specific protein fatty acid translocase - FAT/CD36 in sarcolemma. Either AICAR or physical effort, stimulate AMPK activity in obese and type 2 diabetes mellitus, which can further enhance muscle lipid breakdown and the capacity for ATP generation (by the activation of mitochondrial fatty acid $\beta$-oxidation enzymes) (de Lange et al., 2007; Steinberg \& Kemp, 2009).

Physical effort enhances the production of ROS. ROS is produced continuously in mitochondria and its level is reduced by the activation of AMPK. This process has especially been investigated in smooth muscles, because oxidative stress (e.g. induced by free fatty acids) in patients with a metabolic syndrome contributes to the development of cardiovascular diseases (Steinberg \& Kemp, 2009).

In response to the contraction of a myocyte, interleukin-6 (IL-6) is produced and released. Although AMPK participates in the regulation of IL-6 release from oxidative muscle, IL-6 significantly increases AMPK activity in myotubes as well as enhances fatty acid oxidation and insulin-stimulated glucose uptake.

The effect of adiponectin on AMPK activation is not well known. AMPK is probably indirectly activated by increases in levels of intracellular ATP, but it is known that leptin directly stimulates the $\alpha 2$ and $\beta 2$ catalytic subunits expression of AMPK in skeletal muscle. This occurrence correlates with enhanced fatty acid oxidation and the inhibition of lipogenesis. Such leptin action was reported in healthly individuals, but AMPK in obese individuals was not activated by leptin.

Cytokines may also inhibit AMPK activity - tumor necrosis factor $\alpha$ (TNF $\alpha$ ), which signals through the TNF receptor (TNFR) 1, upregulates protein phosphatase 2C (PP2C) and suppresses AMPK. This in turn suppresses fatty-acid oxidation and increases intramuscular diacylglycerol accumulation in skeletal muscle.

AMPK is a major cellular energy sensor and a master regulator of metabolic homeostasis several processes necessary for the mechanical function of muscles are controlled by 1 enzyme. Today, AMPK is already the target of several classes of drugs used for type 2 diabetes mellitus, including metformin and tiazolidinediones (Steinberg \& Kemp, 2009; Zhang et al., 2009). 


\section{Myocyte modulators and insulin resistance}

Adipose tissue protects other cell tissues from "lipotoxicity", but obesity causes dysregulation of lipolysis and lipid oversupply into muscles and other tissues. Adipose tissue also informs the brain and other tissues about this dysregulation through adiponectin, leptin, resistin, IL-6 and TNF $\alpha$ (Steinberg \& Kemp, 2009). Not only is the adipose tissue recognized as an endocrine organ, it also produces modulators relevant for IR. There is also the hepatic insulin sensitizing substance (HISS), which after being released from the liver, acts on skeletal muscles to stimulate glucose storage in the form of glycogen. The intramyocytal signaling cascade in type 2 diabetes mellitus is impaired due to insulin modulation by HISS and in the future we will probably differentiate these two types of diabetes mellitus in the GP's surgery (Lautt et al., 2010).

It is clear that insulin activates the pathway of kinase Akt and hampers AMPK activity, but despite the developing knowledge about AMPK, we still do not know enough about IR. When skeletal muscles become unable to switch between glucose and fatty acid use, the cause of glucose uptake and utilization is dramatically lessened.

There is evidence that nitric oxide (NO) is a modulator of insulin action and insulin resistance is linked to inducible NO synthase (iNOS) induction in skeletal muscle cells (and in other insulin target cells as well). An increased expression of multiple inflammatory cytokines (TNF $\alpha$, interferon-c, IL-6, and IL-1 $\beta$ ) observed in obesity has to induce iNOS as it was shown on an animal model of acute systemic inflammation that iNOS in muscle (also in liver and adipose) tissue was induced by the administration of the endotoxin lipopolysaccharide (LPS). Cytokines modulate glucose transport in the skeletal muscle by inducing the expression of iNOS. Moreover, a high-dose of aspirin improves glucose metabolism in patients with type 2 diabetes mellitus. There is also genetic evidence that iNOS mediates skeletal muscle IR in obese high-fat fed mice and that the induction of iNOS in skeletal muscle modulates wholebody glucose metabolism (Cha et al., 2011; Soskić et al., 2011).

AMPK phosphorylates and activates NOS, and physiological levels of NO can stimulate glucose uptake and oxidation in human skeletal muscles, but the meaning of NOS for glucose homeostasis is still cloudy (Steinberg \& Kemp, 2009). Physical effort increases NOS activity and the expression of neuronal (nNOS) and endothelial (eNOS) NOS. There is some evidence that mitochondrial respiration in myocytes is inhibited by NOS activity. NO donors, such as sodium nitroprusside, raise skeletal muscle cGMP content and increase glucose uptake in rats, but the AICAR effect is not blocked by NOS inhibitors (McConell \& Wadley, 2008). Chronic administration of NOS inhibitors (monomethylarginine - L-NAME) in drinking water decreases glucose tolerance in rats (Balon et al., 1999).

Another therapeutic target in insulin resistance may be stearoyl-CoA desaturase (SCD). The major product of SCD - oleic acid, may play an important role in the regulation of intracellular ceramide synthesis, which is recognized as an insulin-desensitizing lipid molecule. The lack of the SCD1 gene increases the rate of fatty acid B-oxidation - it results in a decrease of free fatty acids and long-chain fatty acyl-CoAs content in the soleus and red gastrocnemius SCD1-/- mice models. The AMPK pathway is also involved in this process. It 
increases this enzyme's phosphorylation and contributes to an increase of insulin sensitivity in the muscles. The expressions of a protein-tyrosine phosphatase 1B (PTP-1B) is down regulated in the case of SCD1 deficiency. This expression is responsible for the sustained insulin receptor autophosphorylation (Dobrzyn \& Dobrzyn, 2006; Dobrzyn et al., 2010).

PUFA, cholesterol, vitamin A, hormonal signals (e.g. insulin, glucagon), environmental and developmental factors (temperature changes, metals), alcohol, thiazolinediones, peroxisomal proliferators modulate stearoyl-CoA desaturase (SCD) expression in adipose tissue and liver. It is proven that some of these compounds are able to modulate SCD by catalyzing the synthesis of monounsaturated fatty acids (mainly oleate and palmitoleate acids) in muscles. Glucose is probably the most important modulator of SCD expression in the skeletal muscle. Its longterm oversupply increases SCD expression and enzyme activity - it leads to fast and specific changes in fatty acid metabolism. Further investigations of SCD modulators may be relevant, because stress and inflammatory stimuli can also modulate Transcriptional regulation of SPT, which is the first committed step in de novo ceramide synthesis (Dobrzyn \& Dobrzyn, 2006).

There is also evidence that insulin resistance and type 2 diabetes mellitus are linked to the reduction of insulin receptor substrate (IRS-1) expression, and the impairment of PI3K and kinase Akt activity in skeletal muscle. Impaired insulin-mediated glucose transport is a characteristic defect in type 2 diabetes mellitus. There is normal expression of the human GLUT4 gene in type 2 diabetes mellitus, but a cause of insulin resistance is searched out in traffic of this glucose transporter to sarcolemma. The translocation of GLUT4 to the membrane increases its glucose permeability. GLUT4 intracellular redistribution is directly linked to a signaling protein known as AS160 which may be activated by both the insulin pathway and AMPK. Hampering of the Akt substrate of $160 \mathrm{kDa}$ (AS160), phosphorylation (as a result of Akt phosphorylation in the position of Thr308) diminishes the activity of AS160. Impaired insulin-stimulated phosphorylation of AS160 has been discovered in patients with insulin resistance or/and type 2 diabetes mellitus (Treebak et al., 2007).

When no muscles stimulation exists, then only about $10 \%$ of GLUT- 4 transporters are located in the sarcolemma, but their amount increases to 50\% after 2-3 minutes of stimulation with insulin. Relocation of GLUT4 from an intracellular pool of myocytes to its membrane is phosphorylated and activated not only by insulin (associated with the activation of kinase PI3K), but also may be activated by IGF1, EGF and AICAR. NO induces the upregulation of GLUT4 mRNA (by the AMPK dependent pathway) (Wang et al., 1996; Russell et al., 1999).

The role of NO in a skeletal muscle has still not been investigated enough. The interaction of AMPK, NOS enzymes, and NO levels in skeletal muscles is intriguing (AMPK activates eNOS and nNOS, but AMPK activation inhibits iNOS expression). We think that the significance of the effect of SCD1 deficiency/downregulation on lipid metabolism and insulin sensitivity in the skeletal muscle is underrated, as lipids are transported through sarcolemma (Steinberg \& Kemp, 2009).

The last investigation showed that dysregulation in PA uptake and reduced CD36 protein level in murine myocyte are recognized as a reason of metabolic complications analogous to 
"the cardio-metabolic syndrome" in patients treated with a protease inhibitor of the human immunodeficiency virus (Richmond et al., 2010).

It is a real challenge to establish a cause of diabetes mellitus type 2, which is probably multifactorial. One of those factors is a sedentary lifestyle, another one is obesity and a high fat calorie diet, they induce inflammatory cytokines and reduce insulin signaling. This subsequent loss of homeostatic signaling yields a low heat shock proteins (HSP) state (Atalay et al., 2009). The HSPs respond to a wide variety of stress factors (e.g. elevated temperature, hypoxia, altered $\mathrm{pH}$ ) and first studies in patients with diabetes mellitus type 2 revealed that hot-tub therapy is able to improve glycemic control (Gupte et al., 2009, as cited in Hooper, 1999). Later it was described that HSP90 maintains the Akt and plays an important role in the regulation of glucose transport mediated by insulin (Atalay et al. 2009, as cited in Sato S. et al. 2000). Moreover, a lower level of HSP72 was found in skeletal muscles of patients with type 2 diabetes and some HSPs were strongly induced in skeletal muscle with physical exercises especially in fast-twitch muscles (Atalay et al. 2009, as cited in Bruce, 2003 and Kurucz, 2002). A recent study demonstrated that heat treatment protects skeletal muscles in rats from a high-fat diet-induced insulin resistance and provided strong evidence that HSP induction in skeletal muscles could be a potential therapeutic treatment for obesity-induced insulin resistance (Gupte et al., 2009).

The HSP60 expression improves mitochondrial dysfunction observed in skeletal muscles of patients with type 2 diabetes as measured by oxidative capacity, with a high-fat diet in parallel with increased mitochondrial protein expression (Gupte et al., 2009). Also thiazolidinediones increase expression of an array of mitochondrial proteins in diabetic mousses (it also concerns PGC1 $\alpha$, the master regulation of mitochondrial biogenesis) (Cantó et al., 2009) and we think that mitochondria are a primary target of heat therapy from oxidative stress.

Recently, BGP15 (an HSP inducer) was found as a potent insulin sensitizer, which increases muscle glucose utilization (Literáti-Nagy et al. 2009). It protects the mitochondrial membrane against oxidative damage by the activation of PI-3-kinase - Akt pathway. This well-known cytoprotective pathway is suppressed by Poly (ADP-ribose) polymerase (PARP), which can induce rapid cellular $\mathrm{NAD}^{+}$and $\mathrm{ATP}$ pool depletion leading to mitochondrial dysfunction. The small molecule BGP15 is an inhibitor of PARP presented in eukaryotes and may be a novel strategy for therapies of insulin resistance (Halmosi et al. 2001)

We were able to find more evidence that our surroundings also have input in type 2 diabetes mellitus etiology. Bisphenol-A (BPA), a component of polycarbonate plastics and epoxy resins, widely used in multiple consumer products like plastic food/fluids containers is commonly recognized as a disruptor of glucose homeostasis, which provokes hyperinsulinemia and insulin resistance. The measurable levels of this synthetic estrogen were reported in human serum, urine, breast milk, fetal plasma, and placental tissue. It probably modulates the glucose transporter GLUT4 through two estrogen receptors (ER $\alpha$ and ER $\beta$ ). Studies showed that elevated BPA level was associated with elevated hemoglobin A1c (HbA1c). Also higher urinary BPA level was found to be associated with diabetes type 2 diabetes mellitus independently of traditional diabetes risk factors (Lang et al., 2008; Vandenberg et al., 2007; Li et al., 2012). On the other hand, $\alpha$-lipoic acid (LA) (found abundantly in green vegetables) 
affects the insulin-signaling cascade and protects myocyte from oxidative stress-induced insulin resistance (Maddux et al., 2001). Also green and black teas suppress insulin resistance by retaining the expression of GLUT4 in skeletal muscle. Epidemiological data suggests that drinking green tea is negatively associated with type 2 diabetes (Anderson \& Polansky 2002; Nishiumi et al 2010; Yan et al. 2012). Green tea reduces oxidative stress in animal adipocytes (Yan et al. 2012), it also decrease ROS content of cultured mouse myotubes (Buetler et al., 2002), but we have not yet investigated, if green tea protects myocytes from oxidative stressinduced insulin resistance. Several international prospective studies show a protective effect of coffee on the development of type 2 diabetes (Kempf \& Martin 2010). We focused on the modulators crucial for peripheral insulin resistance, but impaired pancreatic glucose sensing is involved in etiology of diabetes mellitus type 2 as well. It is a genetically complex chronic disease, but lifestyle changes can delay progression to diabetes, even in high-risk individuals, for at least a decade (Cho et al. 2012; Knowler WC et al. 2009).

\section{Muscular contraction - underestimated modulator}

Physical exercise is a potent stimulus for increasing glucose uptake in the complete absence of insulin. This mechanism may be modulated by AMPK, Akt/protein kinase B phosphorylation, $\mathrm{NO}$ and $\mathrm{Ca}^{2+} /$ calmodulin-dependent protein kinase (CaMK) and protein kinase $\mathrm{C}$ (PKC) and is initiated by acute exercise. This is a short-lived effect, which disappears in 16-48 h. However, repeated physical exercise results in an increase in insulin action, sustain in skeletal muscle of patients with insulin resistance and type 2 diabetes mellitus. This improvement in insulin sensitivity is related to increasing AS160 phosphorylation, expression and/or activity protein kinase B (Akt) and AMPK (Hawley \& Lessard, 2008; Ismail-Beigi, 2012; Cahová et al., 2007; Wasserman et al., 2011).

Exercise training results also in glucose uptake through an increase in the level of GLUT4 trafficking. Training may cause increasing 30\% of GLUT4 in sarcolemma of patients with type 2 diabetes mellitus and 20\% of GLUT4 in control group (Wasserman et al., 2011). We outlined above, how muscle contraction my improve insulin sensitivity by increased lipid oxidation. There is also clinical evidence that physical activity (150 min/week) and diet-induced weight loss of $5-7 \%$ are able to reduce the risk of progression from impaired glucose tolerance to type 2 diabetes mellitus. Therefore, very recently the guidance in type 2 diabetes mellitus has been changed - more attention is given to muscle contraction as a modulator, which has become a key part of type 2 diabetes mellitus self-management (Sigal et al., 2006).

\section{Conclusion}

Type 2 diabetes mellitus is still a challenge for biochemists, clinicians and epidemiologists. It has been assessed that 23.6 million people lived in the US in 2007 with diagnosed and undiagnosed diabetes ( $7.8 \%$ of the general population). There are forecasts that 366 million people in the world will live with type 2 diabetes mellitus in 2030 (Zhang et al., 2009).

Recently, physical exercise has been recognized by health organizations as an undoubted modulator of glucose uptake in skeletal muscles. We hope that awareness of this fact will be 
created by promotion in the GP's surgery and through mass media. Muscle contraction as a modulator of GLUT4 expression, which also improves insulin signal transduction at the level of PI3K and AS160, and activates AMPK, is able not only to diminish a dose of oral medicine in type 2 diabetes mellitus, but also to influencethe future of patients with insulin resistance.

\section{Author details}

Mariusz Henryk Madalinski

The Pennine Acute Hospitals NHS Trust, Manchester, UK

Leszek Kalinowski*

Department of Medical Laboratory Diagnostics, Chair of Clinical Chemistry and Biochemistry, Medical University of Gdansk, Gdansk, Poland

\section{Acknowledgement}

This research was supported by the Ministry of Science and Higher Education/the National Science Centre, Poland, and the Foundation for Polish Science.

\section{References}

Anderson RA. \& Polansky MM. (2002). Tea enhances insulin activity. Journal of Agricultural and Food Chemistry. Vol.50, No.24, (November), pp. 7182-7186.

Atalay M., Oksala N., Lappalainen J., Laaksonen DE., Sen CK. \& Roy S. (2009). Heat shock proteins in diabetes and wound healing. Current Protein $\mathcal{E}$ Peptide Science, Vol.10, No.1, (February), pp. 85-95.

Balon TW., Jasman AP. \& Young JC. (1999). Effects of chronic N(omega)nitro-L-arginine methyl ester administration on glucose tolerance and skeletal muscle glucose transport in the rat. Nitric Oxide, Vol.3, No.4, (August), pp. 312-320.

Buetler TM., Renard M., Offord EA., Schneider H. \& Ruegg UT. (2002). Green tea extract decreases muscle necrosis in mdx mice and protects against reactive oxygen species. The American Journal of Clinical Nutrition Vol.75, No.4, (April), pp. 749-53.

Cantó C, Gerhart-Hines Z, Feige JN, Lagouge M, Noriega L, Milne JC, Elliott PJ, Pere Puigserver P. \& Auwerx J. (2009). AMPK regulates energy expenditure by modulating $\mathrm{NAD}^{+}$metabolism and SIRT1 activity. Nature, Vol.458, (April), pp. 1056-1060.

Cahová M., Vavrínková H. \& Kazdová L. (2007). Glucose-fatty acid interaction in skeletal muscle and adipose tissue in insulin resistance. Physiological Research, Vol. 56, No.1, pp. 1-15.

Cantó C, Gerhart-Hines Z, Feige JN, Lagouge M, Noriega L, Milne JC, Elliott PJ, Pere Puigserver P. \& Auwerx J. (2009). AMPK regulates energy expenditure by modulating $\mathrm{NAD}^{+}$metabolism and SIRT1 activity. Nature, Vol.458, (April), pp. 1056-1060.

Cha HN., Song SE., Kim YW., Kim JY., Won KC. \& Park SY. (2011). Lack of inducible nitric oxide synthase prevents lipid-induced skeletal muscle insulin resistance without attenuating cytokine level. Journal of Pharmacological Sciences, Vol.117, No.2, pp. 77-86.

\footnotetext{
${ }^{*}$ Corresponding Author
} 
Cho AH., Killeya-Jones LA., O'Daniel JM., Kawamoto K., Gallagher P., Haga S., Lucas JE., Trujillo GM., Joy SV. \& Ginsburg GS. (2012). Effect of genetic testing for risk of type 2 diabetes mellitus on health behaviors and outcomes: study rationale, development and design. BMC Health Services Research, Vol.12, No.16, (January), pp. 1- 11.

de Lange P., Moreno M., Silvestri E., Lombardi A., Goglia F. \& Lanni A. (2007). Fuel economy in food-deprived skeletal muscle: signaling pathways and regulatory mechanisms. Federation of American Societies for Experimental Biology Journal, Vol.21, No.13, (November), pp. 3431-3441.

Diaz-Sylvester PL., Porta M. \& Copello JA. (2008). Halothane modulation of skeletal muscle ryanodine receptors: dependence on $\mathrm{Ca}^{2+}, \mathrm{Mg}^{2+}$, and ATP. American Journal of Physiology - Cell Physiology, Vol.294, No.4, (April), pp. C1103-C1112.

Dobrzyn A. \& Dobrzyn P. (2006). Stearoyl-CoA desaturase - a new player in skeletal muscle metabolism regulation. Jorunal of Physiology and Pharmacology, Vol. 57 Suppl, No.10, pp. 31-42.

Dobrzyn P., Pyrkowska A., Jazurek M., Szymanski K., Langfort J. \& Dobrzyn A. (2010). Endurance training-induced accumulation of muscle triglycerides is coupled to upregulation of stearoyl-CoA desaturase 1. Journal of Applied Physiology, Vo.109, No.6, (December), pp. 1653-1661.

Eltit JM., Li H., Ward ChW., Molinski T., Pessah IN., Allen PD. \& Lopez JR. (2011). Orthograde dihydropyridine receptor signal regulates ryanodine receptor passive leak. Proceedings of the National Academy of Sciences USA, Vol.108, No.17, (April), pp. 7046-7051.

Farahat WA. \& Herr HM. (2010). Optimal workloop energetics of muscle-actuated systems: an impedance matching view. PLoS Computational Biology, Vol.6, No.6, (June), pp. e1000795.

Feng W., Tu J., Pouliquin P., Cabrales E., Sheng X., Dulhunty A., Worley PF., Allen PD. \& Pessah IN. (2008). Dynamic regulation of ryanodine receptor type 1 (RyR1) channel activity by Homer 1. Cell Calcium, Vol.43, No.3, (August), pp. 307-314.

Gupte AA., Bomhoff GL., Swerdlow RH. \& Geiger PC. (2009). Heat treatment improves glucose tolerance and prevents skeletal muscle insulin resistance in rats fed a high-fat diet. Diabetes. Vol. 58, No.3, (March), pp. 567-578.

Halmosi R., Berente Z., Osz E., Toth K., Literati-Nagy P. \& Sumegi B. (2001). Effect of poly(ADP-ribose) polymerase inhibitors on the ischemia-reperfusion-induced oxidative cell damage and mitochondrial metabolism in Langendorff heart perfusion system. Molecular Pharmacology. Vol. 59, No.6, (June), pp. 1497-1505.

Hawley JA., Hargreaves M. \& Zierath JR. (2006). Signalling mechanisms in skeletal muscle: role in substrate selection and muscle adaptation. Essays in Biochemistry. Vol. 42, pp. 1-12.

Hawley JA. \& Lessard SJ. (2008). Exercise training-induced improvements in insulin action. Acta Physiol ogica (Oxford), Vol.192, No.1, (January), pp. 127-135.

Ismail-Beigi F. (2012). Clinical practice. Glycemic management of type 2 diabetes mellitus. The New England Journal of Medicine, Vol. 366, No.14, (April), pp. 1319-1327.

Kempf K. \& Martin S. (2010). Coffee and diabetes. Medizinische Klinik (Munich). Vol.105, No. 12, (December), pp. 910-915.

Diabetes Prevention Program Research Group, Knowler WC., Fowler SE., Hamman RF., Christophi CA., Hoffman HJ., Brenneman AT., Brown-Friday JO., Goldberg R., Venditti E. \& Nathan DM. (2009). 10-year follow-up of diabetes incidence and weight loss in the Diabetes Prevention Program Outcomes Study. The Lancet, Vol. 374, No. 9702, (November) pp: 1677-1686. 
Lang IA., Galloway TS., Scarlett A., Henley WE., Depledge M., Wallace RB. \& Melzer D. (2008). Association of urinary bisphenol A concentration with medical disorders and laboratory abnormalities in adults. The Journal of the American Medical Association, Vol.300, No.11, (September), pp. 1303-1310.

Lautt WW., Ming Z. \& Legare DJ. (2010). Attenuation of age- and sucrose-induced insulin resistance and syndrome $X$ by a synergistic antioxidant cocktail: the AMIS syndrome and HISS hypothesis. Canadian Journal of Physiology and Pharmacology, Vol. 88, No.3, (March), pp. 313-323.

Lawler JM., Kim JH., Kwak HB. \& Barnes WS. (2010). Redox modulation of diaphragm contractility: Interaction between DHPR and RyR channels. Free Radical Biology $\mathcal{E}$ Medicine, Vol.49, No.12, (December), pp. 1969-1977.

Li Y., Burns KA., Arao Y., Luh CJ. \& Korach KS. (2012). Differential estrogenic actions of endocrine-disrupting chemicals bisphenol A, bisphenol AF and zearalenone through estrogen receptor $\alpha$ and $\beta$ in vitro. Environmental Health Perspectives, (April), [Epub ahead of print].

Lim ChT., Kola B. \& Korbonits M. (2010). AMPK as a mediator of hormonal signaling. Journal of Molecular Endocrinology, Vol.44, No.2, (February), pp. 87-97.

Lira VA., Brown DL., Lira AK., Kavazis AN., Soltow QA., Zeanah EH. \& Criswell DS. (2010). Nitric oxide and AMPK cooperatively regulate PGC-1 $\alpha$ in skeletal muscle cells. Journal of Physiology, Vol.588, No.Pt18, (September), pp. 3551-3566.

Literáti-Nagy B., Kulcsár E., Literáti-Nagy, É., Buday B., Peterfai E., Horvath T., Tory K., Kolonics A., Fleming A., Mandl J. \& Koranyi L. (2009). Improvement of insulin sensitivity by a novel drug, BGP-15, in insulin-resistant patients. A proof of concept randomized double-blind clinical trial. Hormone and Metabolic Research, Vol. 41, No.5, (May), pp. 374-380.

Maddux BA, See W, Lawrence JC Jr, Goldfine AL, Goldfine ID, Evans JL. (2001). Protection against oxidative stress-induced insulin resistance in rat L6 muscle cells by mircomolar concentrations of alpha-lipoic acid. Diabetes. Vol.50, No.2, (February), pp. 404-410.

Mailloux RJ. \& Harper ME. (2010). Glucose regulates enzymatic sources of mitochondrial NADPH in skeletal muscle cells; a novel role for glucose-6-phosphate dehydrogenase. Federation of American Societies for Experimental Biology Journal, Vol.24, No.7, (July), pp. 2495-506.

McConell GK. \& Wadley GD. (2008). Potential role of nitric oxide in contraction-stimulated glucose uptake and mitochondrial biogenesis in skeletal muscle. Clinical and Experimental Pharmacology and Physiology, Vol.35, No.12, (December), pp. 1488-1492.

Nishiumi S., Bessyo H., Kubo M., Aoki Y., Tanaka A., Yoshida K. \& Ashida H. (2010). Green and black tea suppress hyperglycemia and insulin resistance by retaining the expression of glucose transporter 4 in muscle of high-fat diet-fed C57BL/6J mice. Journal of Agricultural and Food Chemistry. Vol. 58, No 24, (December), pp.12916-12923.

Perez CF. (2011). On the footsteps of Triadin and its role in skeletal muscle. World Journal of Biological Chemistry, Vol.2, No.8, (August), pp. 177-183.

Pu J., Peng G., Li L., Na H., Liu Y. \& Liu P. (2011). Palmitic acid acutely stimulates glucose uptake via activation of Akt and ERK1/2 in skeletal muscle cells. The Journal of Lipid Research, Vol.52, No.7, (July), pp. 1319-1327. 
Rafaeloff-Phail R., Ding L., Conner L., Yeh WK., McClure D., Guo H., Emerson K. \& Brooks H. (2004). Biochemical regulation of mammalian AMP-activated protein kinase activity by NAD and NADH. Journal of Biological Chemistry, Vol.279, No.51, (December), pp. 52934-52939.

Richmond SR., Carper MJ., Lei X., Zhang S., Yarasheski KE. \& Ramanadham S. (2010). HIVprotease inhibitors suppress skeletal muscle fatty acid oxidation by reducing CD36 and CPT1 fatty acid transporters. Biochimica et Biophysica Acta, Vol.1801, No.5, (May), pp. 559-566.

Russell RR 3rd., Bergeron R., Shulman GI. \& Young LH. (1999). Translocation of myocardial GLUT-4 and increased glucose uptake through activation of AMPK by AICAR. American Journal of Physiology, Vol.277, No.2Pt 2, (August), pp. H643-649.

Sigal RJ., Kenny GP., Wasserman DH., Castaneda-Sceppa C. \& White RD. (2006). Physical activity/exercise and type 2 diabetes: a consensus statement from the American Diabetes Association. Diabetes Care, Vol.29, No.6, (June), pp.1433-1438.

Soskić SS, Dobutović BD, Sudar EM, Obradović MM, Nikolić DM, Djordjevic JD, Radak DJ, Mikhailidis DP, Isenović ER. (2011). Regulation of Inducible Nitric Oxide Synthase (iNOS) and its Potential Role in Insulin Resistance, Diabetes and Heart Failure. The Open Cardiovascular Medicine Journal, Vol.5, (July), pp. 153-163.

Steinberg GR \& Kemp BE. (2009). AMPK in health and disease. Physiological Review, Vol.89, No.3, (July), pp.1025-1078.

Treebak JT., Birk JB., Rose AJ., Kiens B., Richter EA. \& Wojtaszewski JF. (2007). AS160 phosphorylation is associated with activation of alpha2beta2gamma1- but not alpha2beta2gamma3-AMPK trimeric complex in skeletal muscle during exercise in humans. American Journal of Physiology - Endocrinology and Metabolism, Vol.292, No.3, (March), pp. E715-722.

Wang W., Hansen PA., Marshall BA., Holloszy JO. \& Mueckler M. (1996). Insulin unmasks a $\mathrm{COOH}$-terminal Glut4 epitope and increases glucose transport across T-tubules in skeletal muscle. Journal of Cell Biology, Vol.135, No.2, (October), pp.415-430.

Vandenberg LN., Hauser R., Marcus M., Olea N. \& Welshons WV. (2007). Human exposure to bisphenol A (BPA). Reproductive Toxicology, Vol.24, No.2, (August-September), pp.139-177.

Verhoeven AJ., Woods A., Brennan CH., Hawley SA., Hardie DG., Scott J., Beri RK. \& Carling D. (1995). The AMP-activated protein kinase gene is highly expressed in rat skeletal muscle. Alternative splicing and tissue distribution of the mRNA. European Journal of Biochemistry, Vol.228, No.2, (March), pp.236-243.

Yan J., Zhao Y., Suo S., Liu Y. \& Zhao B. (2012). Green tea catechins ameliorate adipose insulin resistance by improving oxidative stress. Free Radical Biology and Medicine, Vol.52, No.9, (May), pp.1648-1657.

Ye Y., Yaeger D., Owen LJ., Escobedo JO., Wang J., Singer JD., Strongin RM. \& Abramson JJ. (2012). Designing calcium release channel inhibitors with enhanced electron donor properties: stabilizing the closed state of ryanodine receptor type 1. Molecular Pharmacology, Vol.81, No.1, (January), pp. 53-62.

Zhang BB., Zhou G. \& Li C. (2009). AMPK: an emerging drug target for diabetes and the metabolic syndrome. Cell Metabolism, Vol.9, No.5, (May), pp. 407-416. 